\title{
SMOOTHING PROPERTIES IN MULTISTEP BACKWARD DIFFERENCE METHOD AND TIME DERIVATIVE APPROXIMATION FOR LINEAR PARABOLIC EQUATIONS
}

YUBIN YAN

Received 11 May 2004 and in revised form 6 December 2004

A smoothing property in multistep backward difference method for a linear parabolic problem in Hilbert space has been proved, where the operator is selfadjoint, positive definite with compact inverse. By using the solutions computed by a multistep backward difference method for the parabolic problem, we introduce an approximation scheme for time derivative. The nonsmooth data error estimate for the approximation of time derivative has been obtained.

\section{Introduction}

Consider the nonhomogeneous linear parabolic equation

$$
u_{t}+A u=f, \quad \text { for } t>0, \text { with } u(0)=v \text {, }
$$

in a Hilbert space $H$ with norm $\|\cdot\|$, inner product $(\cdot, \cdot)$, where $u_{t}=d u / d t$ and $A$ is a linear, selfadjoint, positive definite, not necessarily bounded operator with a compact inverse, densely defined in $\mathscr{D}(A) \subset H$, where $v \in H$ and $f$ is a function of $t$ with values in $H$.

Since $A^{-1}$ is compact, we assume that $A$ has eigenvalues $\left\{\lambda_{j}\right\}_{j=1}^{\infty}$ and a corresponding basis of orthonormal eigenfunctions $\left\{\varphi_{j}\right\}_{j=1}^{\infty}$. For any arbitrary function $g(\lambda)$, defined on the spectrum $\sigma(A)=\left\{\lambda_{j}\right\}_{j=1}^{\infty}$ of $A$, the operator norm of $g(A)$ can be computed by

$$
\|g(A)\|=\sup _{j}\left|g\left(\lambda_{j}\right)\right|=\sup _{\lambda \in \sigma(A)}|g(\lambda)|
$$

which will be used frequently in this paper.

Let $U^{n}, n \geq 0$, be an approximation of the solution $u\left(t_{n}\right)$ of (1.1) at time $t_{n}=n k$, where $k$ is the time step. We introduce the backward difference operator $\bar{\partial}_{p}, p \geq 1$, by

$$
\bar{\partial}_{p} U^{n}=\sum_{j=1}^{p} \frac{k^{j-1}}{j} \bar{\partial}^{j} U^{n}, \quad \text { where } \bar{\partial} U^{n}=\frac{U^{n}-U^{n-1}}{k} .
$$


It is easy to see that, for any smooth real-valued function $u$,

$$
u_{t}\left(t_{n}\right)=\bar{\partial}_{p} u^{n}+O\left(k^{p}\right), \quad \text { as } k \rightarrow 0, \text { with } u^{n}=u\left(t_{n}\right) .
$$

With $U^{0}, \ldots, U^{p-1}$ given, we define our approximate solution $U^{n}$ by

$$
\bar{\partial}_{p} U^{n}+A U^{n}=f^{n}, \quad \text { for } n \geq p \text {, where } f^{n}=f\left(t_{n}\right) .
$$

It is well known from the theory for numerical solution of ordinary differential equations, see, for example, Hairer and Wanner [4], that this method is $A(\theta)$-stable for some $\theta=\theta_{p}>0$ when $p \leq 6$. The theory of stability and error estimates for the approximation of the solution of (1.1) by a multistep method in both constant and variable time-step cases have been well developed, see Becker [1], Bramble et al. [2], Crouzeix [3], Hansbo [6], LeRoux [7, 8], Palencia and Garcia-Archilla [9], Savaré [10], and Thomée [11], and the references therein.

The purpose of this paper is to consider the smoothing property in multistep backward difference method and time derivative approximation of (1.1). The similar results in single-step methods for homogeneous parabolic problems in general Banach space have been studied, for example, by Hansbo $[5,6]$ and Yan $[12,13]$.

We obtain, in Theorem 2.1, the following smoothing property in multistep backward difference method: if $U^{n}$ is the solution of (1.5) with $f=0$, then we have, with $p \leq 6$,

$$
\left\|\bar{\partial}_{p} U^{n}\right\| \leq C t_{n}^{-1} \sum_{j=0}^{p-1}\left\|U^{j}\right\|, \quad \text { for } n \geq 2 p, U^{0}, U^{1}, \ldots, U^{p-1} \in H
$$

We introduce the norm $|v|_{s}=\left(A^{s} v, v\right)^{1 / 2}, s \in \mathbb{R}$, defined by

$$
|v|_{s}^{2}=\sum_{j=1}^{\infty} \lambda_{j}^{s}\left(v, \varphi_{j}\right)^{2}<\infty, \quad \text { for } s \in \mathbb{R}
$$

where $\left\{\lambda_{j}, \varphi_{j}\right\}_{j=1}^{\infty}$ is the eigensystem of the operator $A$. We see that $|\cdot|_{0}=\|\cdot\|$.

It is natural to approximate the time derivative $u_{t}\left(t_{n}\right)$ of the solution of (1.1) by $\bar{\partial}_{p} U^{n}(n \geq 2 p)$, where $U^{n}, n \geq p$, is computed by the multistep backward difference method (1.5). Approximating $u_{t}\left(t_{n}\right)$ by $\bar{\partial}_{p} U^{n}$, we obtain, in Theorem 3.3, with $n \geq 2 p$,

$$
t_{n}^{2 p+2}\left\|\bar{\partial}_{p} U^{n}-u_{t}\left(t_{n}\right)\right\|^{2} \leq C \sum_{j=p}^{2 p-1}\left(\left|U^{j}-u^{j}\right|_{-2 p}^{2}+k^{2 p+2}\left\|A\left(U^{j}-u^{j}\right)\right\|^{2}\right)+C k^{2 p} G(u)
$$

where

$$
G(u)=\int_{0}^{t_{n}}\left(\left|u^{(p+1)}(s)\right|_{-2 p-1}^{2}+s^{2 p+2}\left|u^{(p+1)}(s)\right|_{1}^{2}+s^{2}\left|u_{t}(s)\right|_{1}^{2}\right) d s+t_{2 p}^{3}\left|u_{t}\left(t_{2 p}\right)\right|_{1}^{2} .
$$

In the case of $f \equiv 0$, if the discrete initial values satisfy, with $U^{0}=v$,

$$
\left|U^{j}-u^{j}\right|_{-2 p}+k^{p+1}\left\|A\left(U^{j}-u^{j}\right)\right\| \leq C k^{p}\|v\|, \quad \text { for } p \leq j \leq 2 p-1, v \in H,
$$


for some suitable discrete starting values $U^{0}, U^{1}, \ldots, U^{p-1}$, then, in Corollary 3.4, we get

$$
\left\|\bar{\partial}_{p} U^{n}-u_{t}\left(t_{n}\right)\right\| \leq C k^{p} t_{n}^{-p-1}\|v\|, \quad \text { for } n \geq 2 p .
$$

We also discuss the starting value approximation in the case of $p=2$. For suitable initial approximation $U^{0}, U^{1}$, we can prove, with $U^{0}=v \in H$,

$$
\sum_{j=2}^{3}\left(\left|U^{j}-u^{j}\right|_{-4}+k^{3}\left\|A\left(U^{j}-u^{j}\right)\right\|\right) \leq C k^{2}\left(\|v\|+\|f(0)\|+\int_{0}^{t_{j}}\left\|f^{\prime}(\tau)\right\| d \tau\right) .
$$

Thus, in the case of $p=2$, our error estimate reads, with $U^{0}=v \in H$,

$$
\left\|\bar{\partial}_{2} U^{n}-u_{t}\left(t_{n}\right)\right\| \leq C k^{2} t_{n}^{-3}\left(\|v\|+\|f(0)\|+\int_{0}^{t_{3}}\left\|f^{\prime}(\tau)\right\| d \tau+K(u)\right), \quad n \geq 4,
$$

where

$$
K(u)^{2}=\int_{0}^{t_{n}}\left(\left|u^{(3)}(s)\right|_{-5}^{2}+s^{6}\left|u^{(3)}(s)\right|_{1}^{2}+s^{2}\left|u_{t}(s)\right|_{1}^{2}\right) d s+t_{4}^{3}\left|u_{t}\left(t_{4}\right)\right|_{1}^{2} .
$$

By $C$ and $c$ we denote large and small positive constants independent of the functions and parameters concerned, but not necessarily the same at different occurrences. When necessary for clarity, we distinguish constants by subscripts.

\section{Smoothing properties}

In this section, we will show the smoothing properties for the multistep backward difference method. Before showing this, we first discuss some properties of the backward difference operator $\bar{\partial}_{p}$ defined by (1.3). We first note that (1.3) can be written in another form as

$$
\bar{\partial}_{p} U^{n}=k^{-1} \sum_{\nu=0}^{p} c_{\nu} U^{n-\nu}
$$

where the coefficients $c_{\nu}$ are independent of $k$. Introducing $P(x)=\sum_{\nu=0}^{p} c_{\nu} x^{\nu}$, it is easy to check that (1.4) is equivalent to

$$
P\left(e^{-\lambda}\right)-\lambda=O\left(\lambda^{p+1}\right), \quad \text { as } \lambda \longrightarrow 0
$$

In fact, with $u(t)=e^{t}$ in (1.4), we have

$$
P\left(e^{-k}\right)-k=O\left(k^{p+1}\right), \quad \text { as } k \longrightarrow 0,
$$

replacing $k$ by $\lambda$, we show (2.2). On the other hand, if (2.2) holds, (1.4) follows from Taylor expansion of $\bar{\partial}_{p} u^{n}$ at $t_{n}$. 
526 Smoothing properties for linear parabolic equations

For $p=1,(1.5)$ reduces to the backward Euler method

$$
\frac{U^{n}-U^{n-1}}{k}+A U^{n}=f^{n}, \quad \text { for } n \geq 1,
$$

and the starting value is $U^{0}=v$.

For $p=2$, we have

$$
\frac{\left((3 / 2) U^{n}-2 U^{n-1}+(1 / 2) U^{n-2}\right)}{k}+A U^{n}=f^{n}, \quad \text { for } n \geq 2,
$$

and both $U^{0}$ and $U^{1}$ are needed to start the procedure.

Bramble et al. [2] obtain the following stability result, that is, with $U^{n}$ the solution of (1.5),

$$
\left\|U^{n}\right\| \leq C \sum_{j=0}^{p-1}\left\|U^{j}\right\|+C k \sum_{j=p}^{n}\left\|f^{j}\right\|, \quad \text { for } n \geq p .
$$

In this paper, we first show the smoothing property for the multistep backward difference method.

Theorem 2.1. Let $p \leq 6$. Then there is a constant $C$, independent of the positive definite operator $A$, such that for the solution $U^{n}$ of (1.5) with $f=0$,

$$
\left\|\bar{\partial}_{p} U^{n}\right\| \leq C t_{n}^{-1} \sum_{j=0}^{p-1}\left\|U^{j}\right\|, \quad \text { for } n \geq 2 p .
$$

To prove this theorem, we need the following lemma from Thomée [11, Lemma 10.3]. LEMMA 2.2. The solution of (1.5) may be written, with $f=0$, as

$$
U^{n}=\sum_{s=0}^{p-1} \beta_{n s}(k A) U^{s}, \quad \text { for } n \geq p
$$

where with $\lambda>0, P(\zeta)=\sum_{\nu=0}^{p} c_{\nu} \zeta^{\nu}$, the $\beta_{n s}(\lambda)$ are defined by,

$$
\beta_{n s}(\lambda)=\sum_{j=p-s}^{p} \beta_{n-s-j}(\lambda) c_{j}, \quad \sum_{j=0}^{\infty} \beta_{j}(\lambda) \zeta^{j}:=(P(\zeta)+\lambda)^{-1}
$$

where it is assumed that $\beta_{n-s-j}(\lambda)=0$ in the case $n-s-j<0$.

If $p \leq 6$, there are positive constants $c, C$, and $\lambda_{0}$ such that

$$
\left|\beta_{j}(\lambda)\right| \leq \begin{cases}C e^{-c j \lambda}, & \text { for } 0<\lambda \leq \lambda_{0} \\ C \lambda^{-1} e^{-c j}, & \text { for } \lambda \geq \lambda_{0}\end{cases}
$$


Proof of Theorem 2.1. By (2.8) and (2.1), we find that

$$
\bar{\partial}_{p} U^{n}=k^{-1} \sum_{\nu=0}^{p} c_{\nu} \sum_{s=0}^{p-1} \beta_{(n-v) s}(k A) U^{s}=\sum_{s=0}^{p-1}\left[k^{-1} \sum_{\nu=0}^{p} c_{\nu} \beta_{(n-v) s}(k A)\right] U^{s} .
$$

By (2.8), we see that $n-v \geq p$ for any $0 \leq v \leq p$, which implies that $n$ must be larger than or equal to $2 p$, that is, $n \geq 2 p$. Since $\bar{\partial}_{p} U^{n}$ is linearly dependent on $U^{s}(0 \leq s \leq p-1)$, it suffices to consider separately the cases when $U^{l} \neq 0,0 \leq l \leq p-1$, and $U^{s}=0,0 \leq$ $s \leq p-1, s \neq l$. In other words, we need to show, for $U^{l} \neq 0,0 \leq l \leq p-1$, and $U^{s}=0$, $0 \leq s \leq p-1, s \neq l$,

$$
\left\|\bar{\partial}_{p} U^{n}\right\| \leq C t_{n}^{-1}\left\|U^{l}\right\|
$$

We first consider the case $0<l \leq p-1$. By Lemma 2.2, we have

$$
\begin{aligned}
\bar{\partial}_{p} U^{n} & =k^{-1} \sum_{\nu=0}^{p} c_{\nu}\left(\beta_{(n-v) l} U^{l}\right) \\
& =k^{-1} \sum_{\nu=0}^{p} c_{\nu}\left(\sum_{j=p-l}^{p} \beta_{n-\nu-l-j}(k A) c_{j}\right) U^{l} \\
& =k^{-1} \sum_{j=p-l}^{p}\left(\sum_{\nu=0}^{p} c_{\nu} \beta_{n-\gamma-l-j}(k A)\right) c_{j} U^{l}, \quad \text { for } 0<l \leq p-1 .
\end{aligned}
$$

We remark that $n-v-l-j$ may be negative. In this case, we assume that $\beta_{n-\nu-l-j}(\lambda)=0$. By Lemma 2.3, we have

$$
\sum_{\nu=0}^{p} c_{\nu} \beta_{n-\nu-l-j}(\lambda)=-\lambda \beta_{n-l-j}(\lambda)
$$

Thus

$$
\bar{\partial}_{p} U^{n}=k^{-1} \sum_{j=p-l}^{p}\left(-k A \beta_{n-l-j}(k A)\right) c_{j} U^{l}
$$

and (2.12) will follow from

$$
\left\|k^{-1} \sum_{j=p-l}^{p}\left(-k A \beta_{n-l-j}(k A)\right) c_{j}\right\| \leq C t_{n}^{-1} .
$$

In view of (1.2), (2.16) may be written as, for $0<l \leq p-1$,

$$
\left|n \lambda \sum_{j=p-l}^{p} \beta_{n-l-j}(\lambda)\right| \leq C, \quad \text { for } \lambda \in \sigma(k A) .
$$


528 Smoothing properties for linear parabolic equations

Now we show (2.17). By (2.10). We have, for small $\lambda, 0 \leq \lambda \leq \lambda_{0}$,

$$
\left|n \lambda \sum_{j=p-l}^{p} \beta_{n-l-j}(\lambda)\right| \leq C \sum_{j=p-l}^{p} n \lambda e^{-c(n-l-j) \lambda} \leq C,
$$

and, for large $\lambda, \lambda>\lambda_{0}$,

$$
\left|n \lambda \sum_{j=p-l}^{p} \beta_{n-l-j}(\lambda)\right| \leq C \sum_{j=p-l}^{p} n e^{-c(n-l-j)} \leq C, \quad \text { for } \lambda \geq \lambda_{0} .
$$

Hence (2.17) holds.

It remains to consider the case $l=0$. We have, by Lemma 2.2,

$$
\bar{\partial}_{p} U^{n}=k^{-1} \sum_{\nu=0}^{p} c_{\nu}\left(\beta_{(n-v) 0} U^{0}\right)=k^{-1}\left(\sum_{\nu=0}^{p} c_{\nu} \beta_{n-\nu-p}(k A)\right) c_{p} U^{0} .
$$

Thus in this case, (2.12) will follow from

$$
\left\|k^{-1} \sum_{\nu=0}^{p}\left(c_{\nu} \beta_{n-\nu-p}(k A)\right) c_{p}\right\| \leq C t_{n}^{-1}
$$

By (1.2), (2.21) may be written as

$$
n\left|\sum_{\nu=0}^{p} c_{\nu} \beta_{n-\nu-p}(\lambda)\right| \leq C, \quad \text { for } \lambda \in \sigma(k A), n \geq 2 p,
$$

or, using (2.14),

$$
\left|n \lambda \beta_{n-p}(\lambda)\right| \leq C, \quad \text { for } \lambda \in \sigma(k A), n \geq 2 p
$$

which we will now prove. For small $0 \leq \lambda<\lambda_{0}$, we have, by (2.10),

$$
\left|n \lambda \beta_{n-p}(\lambda)\right| \leq\left(n \lambda e^{-c n \lambda}\right) e^{c p \lambda} \leq C .
$$

For $\lambda>\lambda_{0}$, using again (2.10), we have,

$$
\left|n \lambda \beta_{n-p}(\lambda)\right| \leq C\left(n e^{-c n}\right) e^{c p} \leq C .
$$

Thus (2.23) holds. Together these estimates complete the proof of Theorem 2.1.

Lemma 2.3. Let $p \leq 6$. Let $\beta_{m}(\lambda), m \geq 0$, and $c_{m}, 0 \leq m \leq p$, be defined as in Lemma 2.2. Then, for any $n \geq 2 p, 0<l \leq p-1, p-l \leq j \leq p$,

$$
\left(c_{0}+\lambda\right) \beta_{n-l-j}(\lambda)+c_{1} \beta_{n-l-j-1}(\lambda)+\cdots+c_{p} \beta_{n-l-j-p}(\lambda)=0,
$$

where it is assumed that $\beta_{n-l-j-v}(\lambda)=0$ in the case of $n-l-j-\nu<0$ for $0 \leq \nu \leq p$. 
Proof. By (2.9), we have

$$
(P(\zeta)+\lambda)\left(\sum_{j=0}^{\infty} \beta_{j}(\lambda) \zeta^{j}\right)=1,
$$

that is,

$$
\left(c_{0}+\lambda+c_{1} \zeta^{1}+c_{2} \zeta^{2}+\cdots\right)\left(\beta_{0}(\lambda)+\beta_{1}(\lambda) \zeta+\beta_{2}(\lambda) \zeta^{2}+\cdots\right)=1 .
$$

Comparing with the coefficients of $\zeta^{j}, j=0,1,2, \ldots$, we get

$$
\begin{gathered}
\left(c_{0}+\lambda\right) \beta_{0}(\lambda)=1, \\
\left(c_{0}+\lambda\right) \beta_{1}(\lambda)+c_{1} \beta_{0}(\lambda)=0, \\
\vdots \\
\left(c_{0}+\lambda\right) \beta_{p}(\lambda)+c_{1} \beta_{p-1}(\lambda)+\cdots+c_{p} \beta_{0}(\lambda)=0, \\
\left(c_{0}+\lambda\right) \beta_{p+1}(\lambda)+c_{1} \beta_{p}(\lambda)+\cdots+c_{p} \beta_{1}(\lambda)=0,
\end{gathered}
$$

which implies that, for any $m \geq 1($ not $m=0)$,

$$
\left(c_{0}+\lambda\right) \beta_{m}(\lambda)+c_{1} \beta_{m-1}(\lambda)+\cdots+c_{p} \beta_{m-p}(\lambda)=0
$$

where $\beta_{m-v}=0$ if $m-v<0$ for $1 \leq v \leq p$.

Note that $n-l-j \geq 1$ since $n \geq 2 p, 0<l \leq p-1, p-l \leq j \leq p$. Thus we obtain (2.26) by replacing $m$ with $n-l-j$ in (2.30). The proof is complete.

\section{Error estimates}

In this section, we will show the error estimates for the approximation $\bar{\partial}_{p} U^{n}$ of the time derivative $u_{t}\left(t_{n}\right)$ in nonsmooth data cases.

We first recall the following stability result, see Thomée [11, Theorem 10.4].

Lemma 3.1. Let $p \leq 6$ and $s \geq 0$, and let $U^{n}$ be the solution of (1.5). Then, with $C$ independent of the positive definite operator $A$,

$$
\begin{aligned}
t_{n}^{s}|| U^{n}||^{2}+k \sum_{j=p}^{n} t_{j}^{s}\left|U^{j}\right|_{1}^{2} \leq & C \sum_{j=0}^{p-1}\left(\left|U^{j}\right|_{-s}^{2}+\left.k^{s}|| U^{j}\right|^{2}\right) \\
& +C k \sum_{j=p}^{n}\left(\left|f^{j}\right|_{-s-1}^{2}+t_{j}^{s}\left|f^{j}\right|_{-1}^{2}\right), \quad \text { for } n \geq p .
\end{aligned}
$$


530 Smoothing properties for linear parabolic equations

By shifting the origin, we have the following generalization of Lemma 3.1.

Lemma 3.2. Let $p \leq 6$ and $s \geq 0$, and let $U^{n}$ be the solution of (1.5). Assume that $m \geq p$ and $U^{m-p}, \ldots, U^{m-1}$ are given. Then, with $C$ independent of the positive definite operator $A$,

$$
\begin{aligned}
t_{n}^{s}|| U^{n} \|^{2}+k \sum_{j=m}^{n} t_{j}^{s}\left|U^{j}\right|_{1}^{2} \leq & C \sum_{j=m-p}^{m-1}\left(\left|U^{j}\right|_{-s}^{2}+\left.k^{s}|| U^{j}\right|^{2}\right) \\
& +C k \sum_{j=m}^{n}\left(\left|f^{j}\right|_{-s-1}^{2}+t_{j}^{s}\left|f^{j}\right|_{-1}^{2}\right), \quad \text { for } n \geq m .
\end{aligned}
$$

We have the following error estimate of time derivative approximation in nonsmooth data case.

Theorem 3.3. Let $p \leq 6$ and let $U^{n}$ and $u$ be the solutions of (1.5) and (1.1), respectively. Then, with $n \geq 2 p$,

$$
t_{n}^{2 p+2}\left\|\bar{\partial}_{p} U^{n}-u_{t}\left(t_{n}\right)\right\|^{2} \leq C \sum_{j=p}^{2 p-1}\left(\left|U^{j}-u^{j}\right|_{-2 p}^{2}+k^{2 p+2}\left\|A\left(U^{j}-u^{j}\right)\right\|^{2}\right)+C k^{2 p} G(u),
$$

where

$$
G(u)=\int_{0}^{t_{n}}\left(\left|u^{(p+1)}(s)\right|_{-2 p-1}^{2}+s^{2 p+2}\left|u^{(p+1)}(s)\right|_{1}^{2}+s^{2}\left|u_{t}(s)\right|_{1}^{2}\right) d s+t_{2 p}^{3}\left|u_{t}\left(t_{2 p}\right)\right|_{1}^{2}
$$

Proof. The error $\varepsilon^{n}=\bar{\partial}_{p} U^{n}-u_{t}\left(t_{n}\right)(n \geq p)$ satisfies

$$
\bar{\partial}_{p} \varepsilon^{n}+A \varepsilon^{n}=-\tau^{n}, \quad \text { where } \tau^{n}=A\left(\bar{\partial}_{p} u\left(t_{n}\right)-u_{t}\left(t_{n}\right)\right) \text {, for } n \geq 2 p \text {. }
$$

Applying Lemma 3.2 with $s=2 p+2, m=2 p$, we have, for $n \geq 2 p$,

$$
t_{n}^{2 p+2}\left\|\varepsilon^{n}\right\|^{2} \leq C \sum_{j=p}^{2 p-1}\left(\left|\varepsilon^{j}\right|_{-2 p-2}^{2}+k^{2 p+2}\left\|\mathcal{\varepsilon}^{j}\right\|^{2}\right)+C k \sum_{j=2 p}^{n}\left(\left|\tau^{j}\right|_{-2 p-3}^{2}+t_{j}^{2 p+2}\left|\tau^{j}\right|_{-1}^{2}\right) .
$$

We now estimate the term $k \sum_{j=2 p}^{n}\left|\tau^{j}\right|_{-2 p-3}^{2}$. We will show that, with any norm $\|\cdot\|$ in $H$,

$$
\left\|\bar{\partial}_{p} u\left(t_{j}\right)-u_{t}\left(t_{j}\right)\right\| \leq C k^{p-1} \int_{t_{j-p}}^{t_{j}}\left\|u^{(p+1)}(s)\right\| d s, \quad \text { for } j \geq 2 p .
$$

Assuming this, we have

$$
\left|\tau^{j}\right|_{-2 p-3}^{2} \leq C k^{2 p-1} \int_{t_{j-p}}^{t_{j}}\left|u^{(p+1)}(s)\right|_{-2 p-1}^{2} d s, \quad \text { for } j \geq 2 p .
$$


Thus

$$
\begin{aligned}
k \sum_{j=2 p}^{n}\left|\tau^{j}\right|_{-2 p-3}^{2} & \leq C k^{2 p} \sum_{j=2 p}^{n} \int_{t_{j-p}}^{t_{j}}\left|u^{(p+1)}(s)\right|_{-2 p-1}^{2} d s \\
& \leq C k^{2 p} \int_{0}^{t_{n}}\left|u^{(p+1)}(s)\right|_{-2 p-1}^{2} d s .
\end{aligned}
$$

It remains to estimate $k \sum_{j=2 p}^{n} t_{j}^{2 p+2}\left|\tau^{j}\right|_{-1}^{2}$. If $j \neq 2 p$, we have, by (3.7) with norm $\left\|A^{1 / 2} \cdot\right\|$,

$$
k \sum_{j=2 p+1}^{n} t_{j}^{2 p+2}\left|\tau^{j}\right|_{-1}^{2} \leq C k^{2 p} \sum_{j=2 p+1}^{n} t_{j}^{2 p+2} \int_{t_{j-p}}^{t_{j}}\left|u^{(p+1)}(s)\right|_{1}^{2} d s .
$$

Here we have $t_{j} \leq c s$ for $s \in\left[t_{j-p}, t_{j}\right], j \geq 2 p+1$, which follows from

$$
t_{j} \leq s \frac{t_{j}}{t_{j-p}} \leq s \frac{t_{2 p+1}}{t_{p+1}} \leq c s, \quad \text { for } j \geq 2 p+1 .
$$

Hence

$$
k \sum_{j=2 p+1}^{n} t_{j}^{2 p+2}\left|\tau^{j}\right|_{-1}^{2} \leq C k^{2 p} \sum_{j=2 p+1}^{n} \int_{t_{j-p}}^{t_{j}} s^{2 p+2}\left|u^{(p+1)}(s)\right|_{1}^{2} d s .
$$

For $j=2 p$, we write, since $\sum_{\nu=0}^{p} c_{v}=0$,

$$
\tau^{2 p}=k^{-1} A\left(\sum_{\nu=0}^{p} c_{\nu} u\left(t_{2 p-\gamma}\right)-u_{t}\left(t_{2 p}\right)\right)=k^{-1} A\left(\sum_{\nu=0}^{p} c_{\nu} \int_{t_{p}}^{t_{2 p-\nu}} u_{t}(s) d s-u_{t}\left(t_{2 p}\right)\right),
$$

and we obtain

$$
k\left|\tau^{2 p}\right|_{-1}^{2} \leq C \int_{t_{p}}^{t_{2 p}}\left|u_{t}(s)\right|_{1}^{2} d s+k\left|u_{t}\left(t_{2 p}\right)\right|_{1}^{2}
$$

which follows from

$$
\begin{aligned}
\left|\tau^{2 p}\right|_{-1}^{2} & \leq C\left(k^{-2} \sum_{\nu=0}^{p}\left|\int_{t_{p}}^{t_{2 p-v}} u_{t}(s) d s\right|_{1}^{2}+\left|u_{t}\left(t_{2 p}\right)\right|_{1}^{2}\right) \\
& \leq C k^{-2} \sum_{\nu=0}^{p}(p k) \int_{t_{p}}^{t_{2 p-v}}\left|u_{t}(s)\right|_{1}^{2} d s+\left|u_{t}\left(t_{2 p}\right)\right|_{1}^{2} \\
& \leq C k^{-1} \int_{t_{p}}^{t_{2 p}}\left|u_{t}(s)\right|_{1}^{2} d s+\left|u_{t}\left(t_{2 p}\right)\right|_{1}^{2} .
\end{aligned}
$$


532 Smoothing properties for linear parabolic equations

Thus, we get

$$
\begin{aligned}
k t_{2 p}^{2 p+2}\left|\tau^{2 p}\right|_{-1}^{2} & \leq C k^{2 p+2}\left(\int_{t_{p}}^{t_{2 p}}\left|u_{t}(s)\right|_{1}^{2} d s+k\left|u_{t}\left(t_{2 p}\right)\right|_{1}^{2}\right) \\
& \leq C k^{2 p}\left(\int_{t_{p}}^{t_{2 p}} s^{2}\left|u_{t}(s)\right|_{1}^{2} d s+t_{2 p}^{3}\left|u_{t}\left(t_{2 p}\right)\right|_{1}^{2}\right) .
\end{aligned}
$$

It remains to estimate (3.7). We write, by Taylor expansion around $t_{j-p}$,

$$
u(t)=\sum_{l=0}^{p} \frac{u^{(l)}\left(t_{j-p}\right)}{l !}\left(t-t_{j-p}\right)^{l}+\frac{1}{p !} \int_{t_{j-p}}^{t}(t-s)^{p} u^{(p+1)}(s) d s \equiv Q(t)+R(t) .
$$

By (1.4) and since $Q(t)$ is a polynomial of degree $p$, we have $\bar{\partial}_{p} Q(t)-Q_{t}(t)=0$. Thus, by $(2.1)$

$$
\bar{\partial}_{p} u\left(t_{j}\right)-u_{t}\left(t_{j}\right)=\bar{\partial}_{p} R\left(t_{j}\right)-R_{t}\left(t_{j}\right)=k^{-1} \sum_{\nu=0}^{p} c_{\nu} R\left(t_{j-\nu}\right)-R_{t}\left(t_{j}\right) .
$$

Noting that

$$
\begin{gathered}
\left\|R\left(t_{j-v}\right)\right\| \leq C k^{p} \int_{t_{j-p}}^{t_{j}}\left\|u^{(p+1)}(s)\right\| d s, \quad \text { for } 0 \leq v \leq p, j \geq 2 p, \\
\left\|R_{t}\left(t_{j}\right)\right\|=\frac{1}{(p-1) !}\left\|\int_{t_{j-p}}^{t_{j}}\left(t_{j}-s\right)^{p-1} u^{(p+1)}(s) d s\right\| \leq C k^{p-1} \int_{t_{j-p}}^{t_{j}}\left\|u^{(p+1)}(s)\right\| d s,
\end{gathered}
$$

we complete the proof of (3.7).

Together these estimates complete the proof.

In the homogeneous case, that is, $f=0$, we have the following nonsmooth data error estimates.

Corollary 3.4. Let $p \leq 6$ and let $U^{n}$ and $u$ be the solutions of (1.5) and (1.1), respectively. Assume that $f=0$ and the discrete initial values satisfy, with $U^{0}=v$,

$$
\left|U^{j}-u^{j}\right|_{-2 p}+k^{p+1}\left\|A\left(U^{j}-u^{j}\right)\right\| \leq C k^{p}\|v\|, \quad \text { for } p \leq j \leq 2 p-1 .
$$

Then, with $C$ independent of the positive definite operator $A$,

$$
\left\|\bar{\partial}_{p} U^{n}-u_{t}\left(t_{n}\right)\right\| \leq C k^{p} t_{n}^{-p-1}\|v\|, \quad \text { for } n \geq 2 p .
$$

Proof. For the solution $u$ of homogeneous parabolic equation, it is easy to show that

$$
\int_{0}^{t_{n}}\left|u^{(p+1)}(s)\right|_{-2 p-1}^{2} d s \leq C\|v\|^{2}, \quad \int_{0}^{t_{n}} s^{2 p+2}\left|u^{(p+1)}(s)\right|_{1}^{2} d s \leq C\|v\|^{2},
$$

and $t_{2 p}^{3}\left|u_{t}\left(t_{2 p}\right)\right|_{1}^{2} \leq C\|v\|^{2}$. Applying Theorem 3.3, we complete the proof. 
Next we will consider the starting value approximation. In Theorem 3.3, we see that it is necessary to define starting approximations $\left\{U^{j}\right\}_{j=0}^{p-1}$ such that

$$
\left|U^{j}-u^{j}\right|_{-2 p}+k^{p+1}\left\|A\left(U^{j}-u^{j}\right)\right\|=O\left(k^{p}\right), \quad \text { for } p \leq j \leq 2 p-1 .
$$

Here we will consider the cases $p=1,2$. The approach can be extended to the general case for $p>2$, but the proof is more complicated.

In the case of $p=1$, the approximate solution is defined by the backward Euler method

$$
\bar{\partial}_{1} U^{n}+A U^{n}=f^{n}, \quad \text { for } n \geq 1 \text {, with } U^{0}=v,
$$

or, with $r(\lambda)=1 /(1+\lambda)$,

$$
U^{n}=r(k A) U^{n-1}+k r(k A) f^{n}, \quad \text { for } n \geq 1 \text {, with } U^{0}=v \text {. }
$$

We then have the following lemma, see Thomée [11, Theorem 9.1].

Lemma 3.5. Let $U^{1}$ and $u$ be the solutions of (3.24) and (1.1), respectively. Then, with $u^{1}=u\left(t_{1}\right), U^{0}=u^{0}=v$,

$$
\begin{aligned}
& \left|U^{1}-u^{1}\right|_{-2}+k^{2}\left\|A\left(U^{1}-u^{1}\right)\right\| \\
& \quad \leq C k\left\|v-A^{-1} f(0)\right\|+C k \int_{0}^{k}\left\|A^{-1} f^{\prime}(\tau)\right\| d \tau+C k^{2} \int_{0}^{k}\left\|f^{\prime}(\tau)\right\| d \tau .
\end{aligned}
$$

In particular, if $f=0$, then

$$
\left|U^{1}-u^{1}\right|_{-2}+k^{2}\left\|A\left(U^{1}-u^{1}\right)\right\| \leq C k\|v\| .
$$

We now turn to the case $p=2$. In this case, we need two starting values $U^{0}$ and $U^{1}$. We will use the backward Euler method to compute $U^{1}$, that is, the approximation $U^{n}$ of the solution $u\left(t_{n}\right)$ of $(1.1)$ is defined by

$$
\bar{\partial}_{2} U^{n}+A U^{n}=f^{n}, \quad \text { for } n \geq 2, \quad \bar{\partial} U^{1}+A U^{1}=f^{1}, \quad \text { with } U^{0}=v .
$$

We have the following lemma.

Lemma 3.6. Let $U^{j}, j=2,3$ and $u$ be the solutions of (3.28) and (1.1), respectively. Then, with $u^{j}=u\left(t_{j}\right), U^{0}=u^{0}=v$,

$$
\left|U^{j}-u^{j}\right|_{-4}+k^{3}\left\|A\left(U^{j}-u^{j}\right)\right\| \leq C k^{2}\left(\|v\|+\|f(0)\|+\int_{0}^{t_{j}}\left\|f^{\prime}(\tau)\right\| d \tau\right), \quad j=2,3 .
$$

In particular, if $f=0$, then

$$
\left|U^{j}-u^{j}\right|_{-4}+k^{3}\left\|A\left(U^{j}-u^{j}\right)\right\| \leq C k^{2}\|v\|, \quad j=2,3 .
$$


534 Smoothing properties for linear parabolic equations

Proof. Here we only prove the case $j=2$, that is, we will show that

$$
\left|U^{2}-u^{2}\right|_{-4}+k^{3}|| A\left(U^{2}-u^{2}\right)||=O\left(k^{2}\right) .
$$

The proof for the case $j=3$ is similar.

Since $\bar{\partial}_{2} U^{2}=k^{-1}\left((3 / 2) U^{2}-2 U^{1}+(1 / 2) U^{0}\right)$, we may write

$$
U^{2}=q_{1}(k A) U^{1}+q_{2}(k A) U^{0}+k P(k A) f^{2},
$$

where

$$
q_{1}(\lambda)=\frac{2}{3 / 2+\lambda}, \quad q_{2}(\lambda)=\frac{-1 / 2}{3 / 2+\lambda}, \quad P(\lambda)=\frac{1}{3 / 2+\lambda} .
$$

Thus, noting that $u^{2}=e^{-2 k A} v+\int_{0}^{2 k} e^{-(2 k-s) A} f(s) d s$, we have

$$
U^{2}-u^{2}=q_{1}(k A)\left(U^{1}-u^{1}\right)+q_{2}(k A)\left(U^{0}-u^{0}\right)+E_{2} .
$$

Here, by simple calculation,

$$
\begin{aligned}
E_{2} & =q_{1}(k A) u^{1}+q_{2}(k A) u^{0}+k P(k A) f^{2}-u^{2} \\
& =Q(k A) v+k b_{0}(k A) f(0)+k R(f),
\end{aligned}
$$

where

$$
\begin{aligned}
Q(\lambda)= & q_{1}(\lambda) e^{-\lambda}+q_{2}(\lambda)-e^{-2 \lambda} \\
b_{0}(\lambda)= & q_{1}(\lambda) \int_{0}^{1} e^{-(1-s) \lambda} d s+P(\lambda)-2 \int_{0}^{1} e^{-2(1-s) \lambda} d s \\
R(f)= & q_{1}(k A) \int_{0}^{1} e^{-(1-s) k A}\left(\int_{0}^{k s} f^{\prime}(\tau) d \tau\right) d s \\
& +P(k A) \int_{0}^{2 k} f^{\prime}(\tau) d \tau-2 \int_{0}^{1} e^{-2(1-s) k A}\left(\int_{0}^{2 k s} f^{\prime}(\tau) d \tau\right) d s .
\end{aligned}
$$

We first show that

$$
k^{3}\left\|A\left(U^{2}-u^{2}\right)\right\| \leq C k^{2}\left(\|v\|+k\|f(0)\|+k \int_{0}^{2 k}\left\|f^{\prime}(\tau)\right\| d \tau\right) .
$$

In fact, noting $U^{0}=u^{0}=v$,

$$
\begin{aligned}
k^{3}\left\|A\left(U^{2}-u^{2}\right)\right\| \leq & k^{3}\left\|A q_{1}(k A)\left(U^{1}-u^{1}\right)\right\|+k^{3}\|A Q(k A) v\| \\
& +k^{3}\left\|k A b_{0}(k A) f(0)\right\|+k^{3}\|k A R(f)\| \\
= & I+I I+I I I+I V .
\end{aligned}
$$

Since $|\lambda Q(\lambda)| \leq C$ and $\left|\lambda b_{0}(\lambda)\right| \leq C$ for $0 \leq \lambda<\infty$, we have

$$
I I \leq C k^{2}\|v\|, \quad I I I \leq C k^{3}\|f(0)\| .
$$


By (3.36), it is easy to see that

$$
I V \leq C k^{3} \int_{0}^{2 k}\left\|f^{\prime}(\tau)\right\| d \tau
$$

Now we turn to $I$. Note that

$$
\begin{aligned}
U^{1}-u^{1}= & \left(r(k A)-e^{-k A}\right) v+k r(k A) f^{1}-\int_{0}^{k} e^{-(k-s) A} f(s) d s \\
= & \left(r(k A)-e^{-k A}\right) v+k r(k A)\left(f(0)+\int_{0}^{k} f^{\prime}(\tau) d \tau\right) \\
& -k \int_{0}^{1} e^{-(1-s) k A}\left(f(0)+\int_{0}^{k s} f^{\prime}(\tau) d \tau\right) d s
\end{aligned}
$$

We find

$$
I=k^{3}\left\|A q_{1}(k A)\left(U^{1}-u^{1}\right)\right\| \leq C k^{2}\left(\|v\|+k\|f(0)\|+k \int_{0}^{k}\left\|f^{\prime}\right\| d \tau\right) .
$$

Combining this with the estimates for $I I, I I I$, and $I V$, we obtain (3.37).

We next show that

$$
\left|A\left(U^{2}-u^{2}\right)\right|_{-6} \leq C k^{2}\left(\|v\|+\int_{0}^{2 k} \| f^{\prime}(\tau)|| d \tau\right) .
$$

As in the proof of (3.37), we write

$$
\begin{aligned}
\left|A\left(U^{2}-u^{2}\right)\right|_{-6} \leq & \left|A q_{1}(k A)\left(U^{1}-u^{1}\right)\right|_{-6}+|A Q(k A) v|_{-6} \\
& +\left|k A b_{0}(k A) f(0)\right|_{-6}+|k A R(f)|_{-6} \\
= & I^{\prime}+I I^{\prime}+I I I^{\prime}+I V^{\prime} .
\end{aligned}
$$

Since $\left|\lambda^{-2} Q(\lambda)\right|<C$ and $\left|\lambda^{-1} b_{0}(\lambda)\right| \leq C$ for $0 \leq \lambda<\infty$, we get

$$
\begin{aligned}
I I^{\prime} & =k^{2}\left\|(k A)^{-2} Q(k A) v\right\| \leq C k^{2}\|v\|, \\
I I I^{\prime} & =k^{2}\left\|(k A)^{-1} b_{0}(k A) A^{-1}\right\| \leq C k^{2}\|f(0)\| .
\end{aligned}
$$

Further, we easily find that

$$
\begin{gathered}
I V^{\prime} \leq C k^{2} \int_{0}^{2 k}\left\|f^{\prime}(\tau)\right\| d \tau \\
I^{\prime} \leq C k^{2}\left(\|v\|+\|f(0)\|+\int_{0}^{k}\left\|f^{\prime}(\tau)\right\| d \tau\right) .
\end{gathered}
$$

Hence (3.43) follows.

Together, these estimates show (3.31). The proof is complete.

Combining Theorem 3.3 with Lemma 3.6, we get the following error estimate in the case $p=2$. 
Corollary 3.7. Let $U^{n}$ and $u$ be the solutions of (3.28) and (1.1), respectively. Then, with $U^{0}=u^{0}=v$,

$$
\left\|\overline{\partial_{2}} U^{n}-u_{t}\left(t_{n}\right)\right\| \leq C k^{2} t_{n}^{-3}\left(\|v\|+\|f(0)\|+\int_{0}^{t_{3}}\left\|f^{\prime}(\tau)\right\| d \tau+K(u)\right), \quad n \geq 4
$$

where

$$
K(u)^{2}=\int_{0}^{t_{n}}\left(\left|u^{(3)}(s)\right|_{-5}^{2}+s^{6}\left|u^{(3)}(s)\right|_{1}^{2}+s^{2}\left|u_{t}(s)\right|_{1}^{2}\right) d s+t_{4}^{3}\left|u_{t}\left(t_{4}\right)\right|_{1}^{2} .
$$

\section{References}

[1] J. Becker, A second order backward difference method with variable steps for a parabolic problem, BIT 38 (1998), no. 4, 644-662.

[2] J. H. Bramble, J. E. Pasciak, P. H. Sammon, and V. Thomée, Incomplete iterations in multistep backward difference methods for parabolic problems with smooth and nonsmooth data, Math. Comp. 52 (1989), no. 186, 339-367.

[3] M. Crouzeix, On multistep approximation of semigroups in Banach spaces, J. Comput. Appl. Math. 20 (1987), no. Special Issue, 25-35.

[4] E. Hairer and G. Wanner, Solving Ordinary Differential Equations. II, Springer Series in Computational Mathematics, vol. 14, Springer, Berlin, 1991, Stiff and differential-algebraic problems.

[5] A. Hansbo, Nonsmooth data error estimates for damped single step methods for parabolic equations in Banach space, Calcolo 36 (1999), no. 2, 75-101.

[6] Strong stability and non-smooth data error estimates for discretizations of linear parabolic problems, BIT 42 (2002), no. 2, 351-379.

[7] M.-N. Le Roux, Semi-discrétisation en temps pour les équations d'évolution paraboliques lorsque l'opérateur dépend du temps, RAIRO Anal. Numér. 13 (1979), no. 2, 119-137.

[8] - Semidiscretization in time for parabolic problems, Math. Comp. 33 (1979), no. 147, 919-931.

[9] C. Palencia and B. García-Archilla, Stability of linear multistep methods for sectorial operators in Banach spaces, Appl. Numer. Math. 12 (1993), no. 6, 503-520.

[10] G. Savaré, $A(\Theta)$-stable approximations of abstract Cauchy problems, J. Numer. Math. 65 (1993), no. 3, 319-335.

[11] V. Thomée, Galerkin Finite Element Methods For Parabolic Problems, Springer Series in Computational Mathematics, vol. 25, Springer, Berlin, 1997.

[12] Y. Yan, Smoothing A properties and approximation of time derivatives for parabolic equations: variable time steps, BIT 43 (2003), no. 3, 647-669.

[13] Smoothing properties and approximation of time derivatives for parabolic equations: constant time steps, IMA J. Numer. Anal. 23 (2003), no. 3, 465-487.

Yubin Yan: Department of Mathematics, The University of Manchester, M13 9PL, Manchester, UK

Current address: Department of Automatic Control and Systems Engineering, Faculty of Engineering, The University of Sheffield, Mappin Street, Sheffield, S1 3JD, UK

E-mail address: y.yan@sheffield.ac.uk 


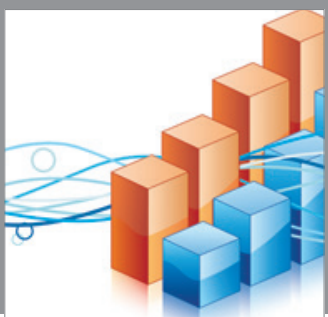

Advances in

Operations Research

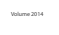

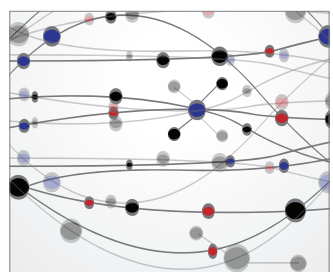

\section{The Scientific} World Journal
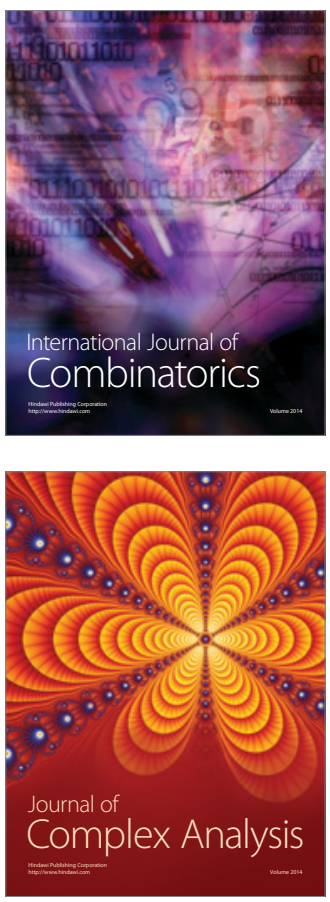

International Journal of

Mathematics and

Mathematical

Sciences
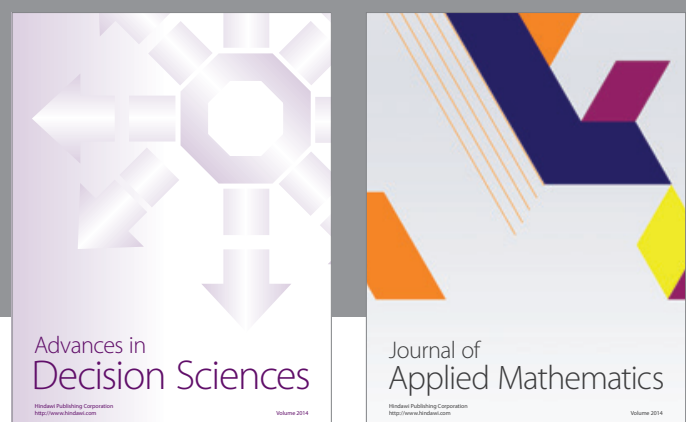

Journal of

Applied Mathematics
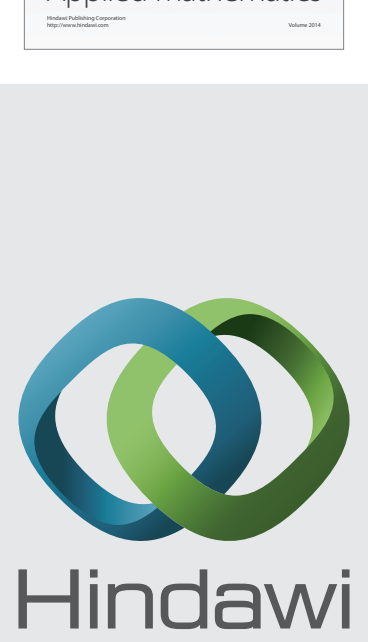

Submit your manuscripts at http://www.hindawi.com
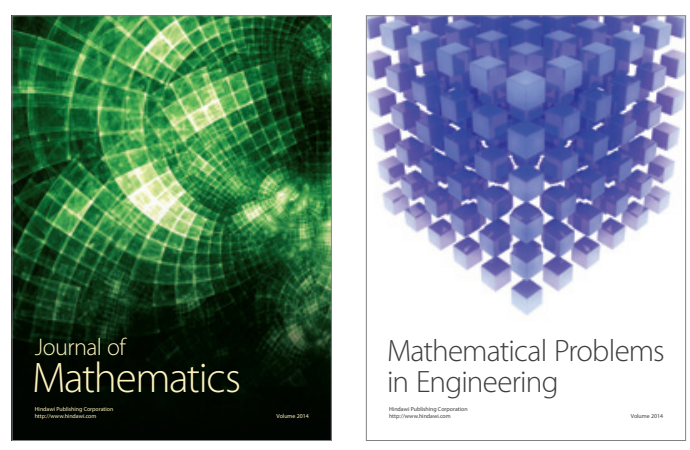

Mathematical Problems in Engineering
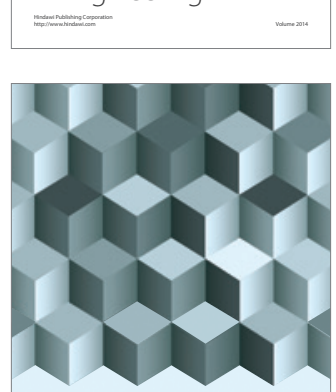

Journal of

Function Spaces
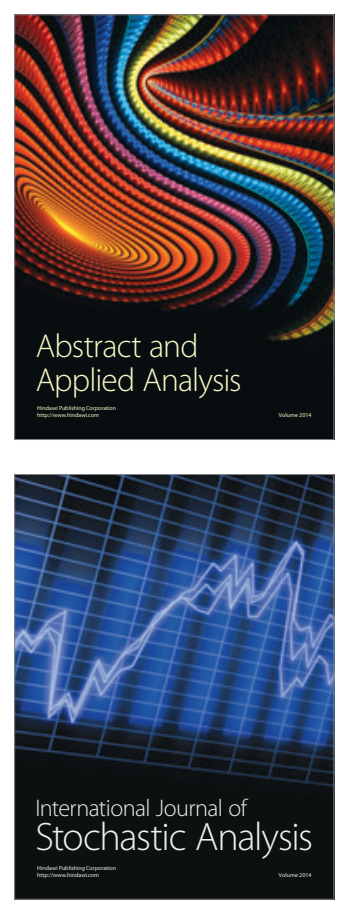

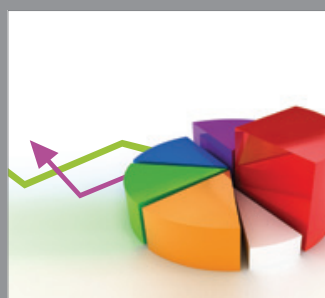

ournal of

Probability and Statistics

Promensencen
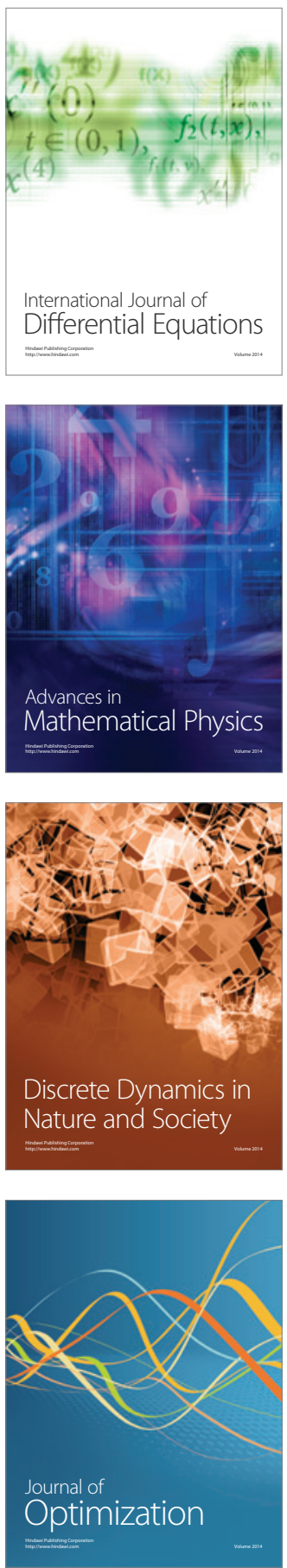